\title{
Morphologic, Distributional, Volumetric, and Intensity Characterization of Periventricular Hyperintensities
}

\author{
M.C. Valdés Hernández, R.J. Piper, M.E. Bastin, N.A. Royle, S. Muñoz Maniega, B.S. Aribisala, C. Murray, I.J. Deary, and J.M. Wardlaw
}

\begin{abstract}
BACKGROUND AND PURPOSE: White matter hyperintensities are characteristic of old age and identifiable on FLAIR and T2-weighted MR imaging. They are typically separated into periventricular or deep categories. It is unclear whether the innermost segment of periventricular white matter hyperintensities is truly abnormal or is imaging artifacts.
\end{abstract}

MATERIALS AND METHODS: We used FLAIR MR imaging from 665 community-dwelling subjects $72-73$ years of age without dementia. Periventricular white matter hyperintensities were visually allocated into 4 categories: 1) thin white line; 2) thick rim; 3) penetrating toward or confluent with deep white matter hyperintensities; and 4) diffuse ill-defined, labeled as "subtle extended periventricular white matter hyperintensities." We measured the maximum intensity and width of the periventricular white matter hyperintensities, mapped all white matter hyperintensities in 3D, and investigated associations between each category and hypertension, stroke, diabetes, hypercholesterolemia, cardiovascular disease, and total white matter hyperintensity volume.

RESULTS: The intensity patterns and morphologic features were different for each periventricular white matter hyperintensity category. Both the widths $(r=0.61, P<.001)$ and intensities $(r=0.51, P<.001)$ correlated with total white matter hyperintensity volume and with each other $(r=0.55, P<.001)$ for all categories with the exception of subtle extended periventricular white matter hyperintensities, largely characterized by evidence of erratic, ill-defined, and fragmented pale white matter hyperintensities (width: $r=0.02, P=.11$; intensity: $r=$ $0.02, P=.84$ ). The prevalence of hypertension, hypercholesterolemia, and neuroradiologic evidence of stroke increased from periventricular white matter hyperintensity categories 1 to 3 . The mean periventricular white matter hyperintensity width was significantly larger in subjects with hypertension (mean difference $=0.5 \mathrm{~mm}, P=.029$ ) or evidence of stroke (mean difference $=1 \mathrm{~mm}, P<.001$ ). $3 \mathrm{D}$ mapping revealed that periventricular white matter hyperintensities were discontinuous with deep white matter hyperintensities in all categories, except only in particular regions in brains with category 3.

CONCLUSIONS: Periventricular white matter hyperintensity intensity levels, distribution, and association with risk factors and disease suggest that in old age, these are true tissue abnormalities and therefore should not be dismissed as artifacts. Dichotomizing periventricular and deep white matter hyperintensities by continuity from the ventricle edge toward the deep white matter is possible.

ABBREVIATIONS: DWMH = deep white matter hyperintensities; IQR = interquartile range; $\mathrm{PVWMH}=$ periventricular white matter hyperintensities; $\mathrm{WMH}=$ white matter hyperintensities

$\mathbf{T}$ he presence of brain white matter hyperintensities is a common neuroradiologic finding in older individuals without disease $^{1}$ and in those with neurologic disease. They appear on CT

Received February 11, 2013; accepted after revision March 21.

From the Brain Research Imaging Centre (M.C.V.H., M.E.B., N.A.R., S.M.M., B.S.A., J.M.W.), College of Medicine and Veterinary Medicine (R.J.P.); Division of Health Sciences (Medical Physics) (M.E.B.); and Department of Psychology (C.M., I.J.D.), University of Edinburgh, Edinburgh, UK.

M.C. Valdés Hernández and R.J. Piper contributed equally to this work.

This work was funded by Age UK and the UK Medical Research Council as part of the project "Disconnected Mind: LBC1936" (http://www.disconnectedmind. ed.ac.uk/); the Centre for Cognitive Ageing and Cognitive Epidemiology (http:// www.ccace.ed.ac.uk/) (G0700704/84698); and the Row Fogo Charitable Trust. Funding from the Biotechnology and Biological Sciences Research Council, the as areas of decreased attenuation. On MR imaging, they appear as areas of increased signal intensity in T2-weighted and FLAIR brain images and as hypointense areas in T1-weighted images. White matter hyperintensities (WMH) are typically separated into periven-

Engineering and Physical Sciences Research Council, and the Economic and Social Research Council is gratefully acknowledged. Imaging was performed in the Brain Research Imaging Centre (http://www.bric.ed.ac.uk/), a Scottish Imaging Network, A Platform for Scientific Excellence Collaboration center.

Paper previously presented at: 36th European Society of Neuroradiology Annual Meeting, September 19-23, 2012; Edinburgh, Scotland.

Please address correspondence to Maria C. Valdés Hernández, MD, Brain Research Imaging Centre, Neuroimaging Sciences, University of Edinburgh, Western General Hospital, Crewe Rd, Edinburgh EH4 2XU, UK; e-mail: mvhernan@staffmail.ed.ac.uk

- Indicates open access to non-subscribers at www.ajnr.org

http://dx.doi.org/10.3174/ajnr.A3612 
tricular or deep categories. ${ }^{2}$ Periventricular white matter hyperintensities (PVWMH) are regarded as hyperintensities adhering to a "continuity rule," so that they are confluent with and extend away from the ventricular wall. ${ }^{2,3}$ Deep white matter hyperintensities (DWMH), conversely, are said to be separated from the PVWMH by at least 1 voxel $^{4}$ and reside within the deep white matter.

It is not clear from previous investigations whether the innermost segment of PVWMH is indeed white matter pathology or is a mere manifestation of artifacts from CSF flow signal. PVWMH that exhibit a regular pattern and are narrower than 2 voxels have been considered true white matter abnormalities by some, ${ }^{2,3}$ whereas others classified them as artifacts. ${ }^{5}$ However, no evidence has been provided to support these conventions.

The criteria for separating WMH into PVWMH or DWMH have typically relied on rules of continuity of $\mathrm{WMH}$ from the lateral ventricles, ${ }^{6,7}$ but distance from the ventricular edges has also been proposed. For example, PVWMH have been reported to penetrate as much as 7 and $13 \mathrm{~mm}$ into the brain parenchyma, ${ }^{8,9}$ and any WMH found within $3 \mathrm{~mm}$ from the ventricular wall have been proposed to belong to a distinct subtype, "juxtaperiventricular." ${ }^{6}$ Some studies ${ }^{2,10}$ render these classifications artificial. Others claim that they are arbitrary ${ }^{11}$ and contrary to pathologic evidence of common vascular mechanisms, or they have suggested that PVWMH and DWMH volumes are highly correlated and have found that their spatial analysis failed to identify distinct subpopulations for PVWMH and DWMH. ${ }^{12}$ Thus, a robust analysis of PVWMH morphology, distribution, and clinical correlates is required to determine a more appropriate classification of these hyperintense regions and their associations.

The purpose of this study was to characterize PVWMH more clearly by their spatial distribution, signal intensity, and relationship to risk factors. The analyses were designed to test 3 hypotheses concerning the definition and classification of PVWMH: 1) A hyperintense thin white line detected along the rim of the lateral ventricle is not a manifestation of a partial volume effect (artifacts) but is evidence of abnormal tissue; 2) PVWMH and DWMH dichotomization is possible and appropriate by a compound rule of continuity from the ventricular surface, unique distribution patterns, and morphologic characteristics; and 3) both PVWMH and DWMH are associated because they have similar distributional properties and common risk factors and therefore are potentially part of the same disease process.

\section{MATERIALS AND METHODS}

\section{Subjects and Brain MR Imaging Acquisition}

Brain axial FLAIR images and self-reported clinical data were obtained from 667 participants of the Lothian Birth Cohort 1936 (353 males and 314 females), a group of community-dwelling

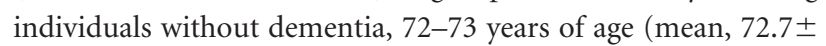
0.7 years) at the time of scanning and data collection. This cohort was recruited as part of the Disconnected Mind project (http:// www.disconnectedmind.ed.ac.uk), an investigation of the aging brain and related cognitive changes. ${ }^{13}$ Formal written consent from all subjects and ethical approval were acquired.

MR imaging was conducted in the Brain Research Imaging Centre, University of Edinburgh (http://www.bric.ed.ac.uk). A Signa Horizon HDx 1.5T clinical scanner (GE Healthcare, Mil- waukee, Wisconsin), equipped with a self-shielding gradient set and a manufacturer-supplied 8-channel phased-array head coil, was used to acquire FLAIR-weighted datasets $(\mathrm{TR} / \mathrm{TE} / \mathrm{TI}=9002 /$ $147.38 / 2200 \mathrm{~ms}$, 4-mm section thickness, matrix $=256 \times 256$, and $15.63-\mathrm{Hz}$ bandwidth) among other sequences. Full details of the MR imaging protocol have been published previously. ${ }^{14}$

\section{Categorization of PVWMH}

We defined 4 categories to describe the morphologic and distributional properties of the PVWMH on the basis of visual rating scales $^{2,15,16}$ and distinctions arising from clinical studies. ${ }^{2,16,17}$ These categories were the following (Fig 1): 1) a thin white line on the borders of the ventricles (corresponding to the commonly termed "pencil-thin" rims $^{2}$ ); 2) a thick rim characterized by attenuated PVWMH with irregular boundaries; and 3) extended PVWMH characterized by either large caps at the horns of the ventricles or wide hyperintense areas that penetrate into the DWMH and can converge with DWMH. These 3 categories are in correspondence with Fazekas visual rating scale PVWMH categories 1, 2, and 3, respectively. An additional category (4) "subtle extended PVWMH" was considered for subjects with extensive evidence of "dirty" or ill-defined, subtle, pale PVWMH ${ }^{18}$ and noncontinuous and diffuse periventricular hyperintensities, with varying and erratic intensity patterns emerging from the lateral ventricle wall, as described previously. ${ }^{10,18,19}$ These were much less well-defined and much less intense than the very obvious white hyperintensities classed in 1-3 above. Each FLAIR image was visually assessed by 2 observers, blinded to each other's results and to any other information (ie, clinical or obtained from image processing or other visual rating scales). Discrepant borderline cases were discussed until agreement was reached. A random subsample ( $n=70)$ was assessed twice by both observers separately; the intraclass correlation coefficient was 0.98 .

\section{Quantitative Measurement of Intracranial and WMH Volumes}

The intracranial volume (ie, contents within the inner skull table including brain tissue, CSF, veins, and dura), with an inferior limit on the axial section just superior to the tip of the odontoid peg at the foramen magnum, was extracted semiautomatically by using the $\mathrm{T}^{*}$-weighted sequence, with the Object Extraction Tool in Analyze (AnalyzeDirect, Mayo Clinic, Rochester, Minnesota) followed by manually editing.

WMH were segmented semiautomatically on the quantized color image obtained after fusing coregistered FLAIR and T2*weighted sequences, mapping them in green and red respectively, and applying minimum variance quantization. This technique is described in Valdés Hernández et al, ${ }^{20}$ validated elsewhere, ${ }^{21}$ and implemented by MCMxxxVI_ALE (a tool freely available from (www.sourceforge.net/projects/bric1936). Intracranial volume was used to correct WMH volumes for head size.

\section{Intensity Analysis}

The intensity of PVWMH was measured by using a tool Intensity Analyzer (freely downloadable from http://www.sourceforge.net/ projects/bric1936). This tool allows a straight line to be plotted on an MR imaging section to generate a uniform readout of the normalized signal-intensity profile along the plotted line ( $y$-axis) ver- 


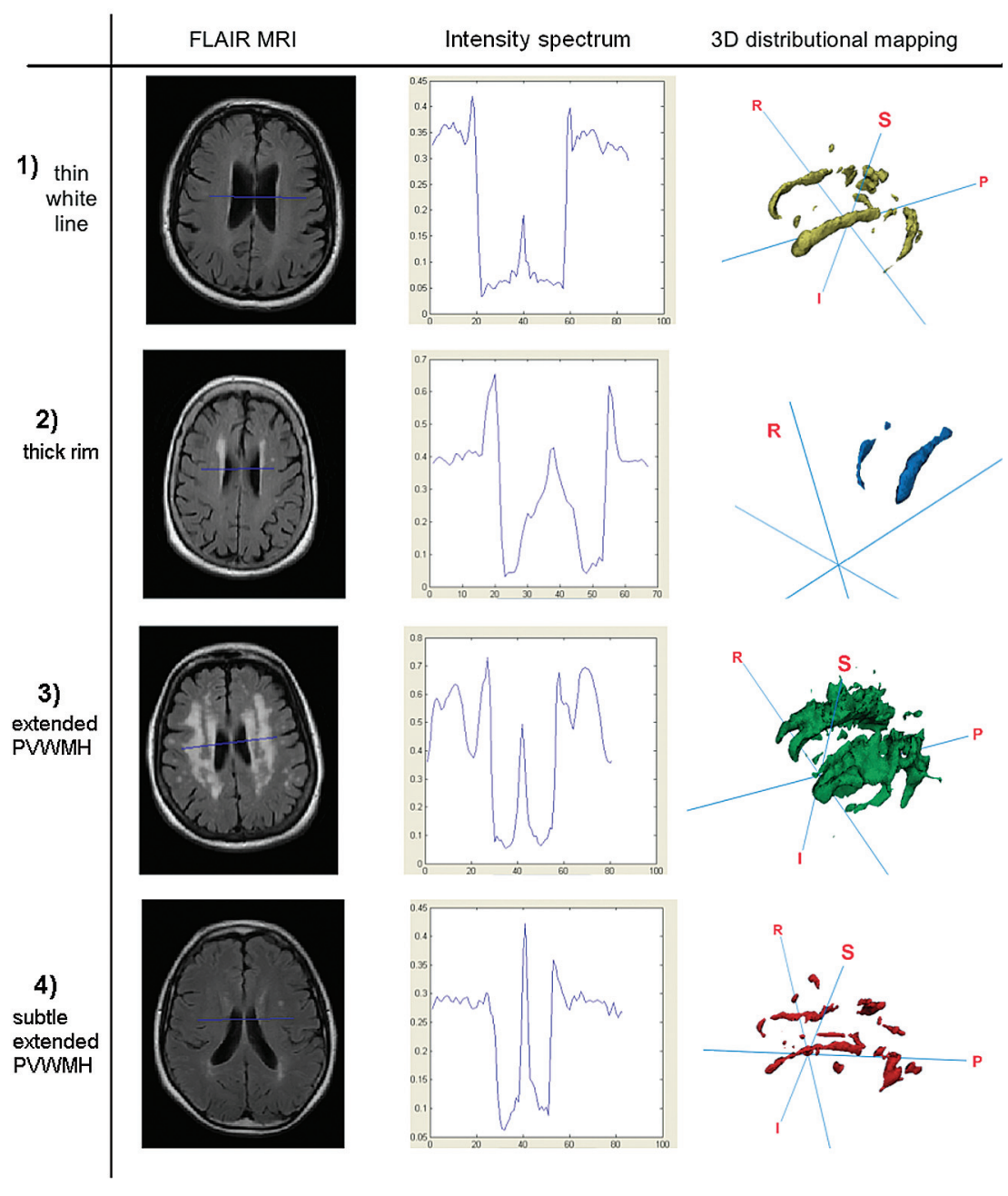

FIG 1. Representative images from each category. From top to bottom: 1) thin white line, 2) thick rim, 3) extended PVWMH, and 4) subtle extended PVWMH. From left to right: axial FLAIR MR imaging, readout from the novel Intensity Analyzer tool, and 3D distributional mapping of $\mathrm{WMH}$. The Intensity Analyzer axes: $\mathrm{x}$, width in millimeters; $\mathrm{y}$, signal intensity (percentage peak from the adjacent lateral trough).

sus its normalized length (x-axis). From each scan, we chose a section toward the top of the lateral ventricles (just after the basal ganglia disappears) and drew a line perpendicular to the interventricular septum starting at the left hemispheric white-gray matter boundary and finishing at the corresponding right boundary, taking care that it crossed the area where the width of the PVWMH was closer to the mean width visually estimated (Fig 1). In all cases, we measured the width of the PVWMH (distance from the edge of the ventricles to the end of the first spike along the plotted line) and their peak intensity (expressed as the percentage increase with respect to the mean intensity of the adjacent lateral trough).

The aforementioned criterion for determining the intensity of the PVWMH was cross-checked as follows: We placed squared regions of interest $(2 \times 2 \mathrm{~mm})$ at the central and innermost region of the hyperintensity rim on 4 consecutive sections by using Analyze 10.0 on a subsample of $10 \%$ of the subjects selected randomly from each PVWMH category and calculated the mean intensity of those regions. The mean intensity of the healthy white matter was calculated by placing a square $(5 \times 5 \mathrm{~mm})$ region of $13.6 \mathrm{~mL})$. interest bilaterally in the frontal white matter (selected for the relative absence of $\mathrm{WMH}){ }^{21}$

\section{D Distributional Visualization of WMH}

3D distributional visualization of $\mathrm{WMH}$ was conducted representing in $3 \mathrm{D}$ the binary masks of the WMH of the random subsample mentioned above by using the surface builder tool in Mango software (University of Texas Health Science Center, Houston, Texas; http://ric.uthscsa. edu/mango). This was to determine whether the spatial separation between PVWMH and DWMH is an artificial convention on the basis of $2 \mathrm{D}$ visualization. ${ }^{12}$

\section{Statistical Analysis}

In addition to the imaging measurements, we analyzed smoking status and clinical data: the presence or absence of hypertension, diabetes, hypercholesterolemia, and cardio- and cerebrovascular disease. Subjects were classified as having cerebrovascular disease if the subject either self-reported stroke or transient ischemic attack or had neuroradiologic evidence of previous stroke on MR imaging, determined as explained elsewhere. ${ }^{20}$

The Kruskal-Wallis test with a post hoc Dunn test was used to identify significant differences between categories and groups of participants with/without the presence of risk factors, and the Spearman test was used to assess the correlations. For all statistical tests, we used GraphPadPrism, Version 5.0 (GraphPad Software, San Diego, California). Statistical significance was set at $P<.05$ (2-tailed). Results are presented as median and interquartile range (IQR) (25\%-75\%) unless stated otherwise.

\section{RESULTS}

\section{Characteristics of the Sample}

In total, $327(49.17 \%)$ subjects had hypertension, 67 (10.08\%) had diabetes (type $1, n=7$ and type $2, n=60), 278(41.80 \%)$ had hypercholesterolemia, and $178(26.77 \%)$ had a history of cardiovascular disease (Table). From the total number of subjects with cerebrovascular disease, $44(6.62 \%)$ self-reported a history of stroke and 95 (14.33\%) had neuroradiologic confirmation of a previous stroke. The median $\mathrm{WMH}$ volume was $7.7 \mathrm{~mL}(\mathrm{IQR}=$

\section{Categorization of PVWMH}

Only 2 subjects did not exhibit any evidence of PVWMH, leaving $665(99.7 \%)$ subjects who were divided into 4 categories as follows: thin white line, 273 (41.05\%); thick rim, 233 (35.04\%); ex- 


\begin{tabular}{|c|c|c|c|c|c|c|c|c|c|c|c|c|c|}
\hline \multirow[b]{2}{*}{ Category of PVWMH } & \multirow{2}{*}{$\begin{array}{c}\text { No. of } \\
\text { Subjects }\end{array}$} & \multicolumn{2}{|c|}{ Smokers } & \multicolumn{2}{|c|}{$\begin{array}{l}\text { Hyper- } \\
\text { tension }\end{array}$} & \multicolumn{2}{|c|}{ Diabetes } & \multicolumn{2}{|c|}{$\begin{array}{c}\text { Hyper- } \\
\text { cholesterolemia }\end{array}$} & \multicolumn{2}{|c|}{$\begin{array}{l}\text { History of } \\
\text { Cardiov. } \\
\text { Disease }\end{array}$} & \multicolumn{2}{|c|}{$\begin{array}{c}\text { History of } \\
\text { Cerebrov. } \\
\text { Disease }\end{array}$} \\
\hline & & No. & $\%$ & No. & $\%$ & No. & $\%$ & No. & $\%$ & No. & $\%$ & No. & $\%$ \\
\hline Thin white line & 273 & 143 & 52.38 & 120 & 43.96 & 30 & 10.99 & 103 & 37.73 & 74 & 27.11 & 32 & 11.72 \\
\hline Thick rim & 233 & 127 & 54.51 & 126 & 54.08 & 26 & 11.16 & 106 & 45.49 & 62 & 26.61 & 44 & 18.88 \\
\hline Extended PVWMH & 90 & 47 & 52.22 & 50 & 55.56 & 6 & 6.67 & 48 & 53.33 & 24 & 26.67 & 30 & 33.33 \\
\hline Subtle extended PVWMH & 69 & 35 & 52.24 & 31 & 46.27 & 5 & 7.46 & 21 & 31.34 & 18 & 26.87 & 9 & 13.43 \\
\hline Total & 665 & 352 & 52.93 & 327 & 49.17 & 67 & 10.08 & 278 & 41.80 & 178 & 26.77 & 115 & 17.29 \\
\hline
\end{tabular}

Note:-Cardiov. Indicates cardiovascular; Cerebrov, cerebrovascular.

\section{WMH load (ml)}

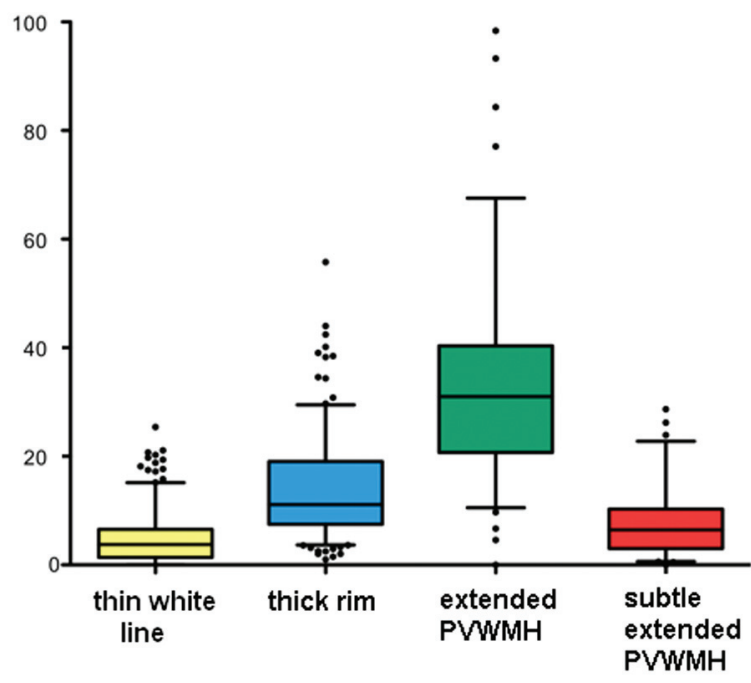

FIG 2. Box-and-whisker plot showing the distribution of PVWMH across the categories. Boxes indicate $25 \%-75 \%$ interquartile range; horizontal lines in boxes, median width; whiskers, 5\%-95\% range; dots, detected outliers that reside outside the whisker range. The distribution of total WMH volumes across these categories is widespread (maximum $=98.38 \mathrm{~mL}$ ), but differences among all categories are statistically significant.

tended PVWMH, 90 (13.53\%); and subtle extended PVWMH, 69 (10.08\%) (Table). The distribution of total WMH volumes across these categories was widespread (from almost none to maximum $=$ $98.38 \mathrm{~mL}$ ), but statistically significant volumetric differences were found among all categories (thin white line median $=3.77 \mathrm{~mL}$, $\mathrm{IQR}=1.39-6.65 \mathrm{~mL}$; thick rim $=11.13 \mathrm{~mL}, \mathrm{IQR}=7.47-19.03$; extended PVWMH $=31.02 \mathrm{~mL}, \mathrm{IQR}=20.73-40.33$; subtle extended PVWMH $=6.46 \mathrm{~mL}, \mathrm{IQR}=3.02-10.26)($ Fig 2).

\section{Intensity Analysis}

The Intensity Analyzer tool identified signature intensity patterns and features for each morphologic category. Figure 1 shows a representative case of each category with the output from the Intensity Analyzer and the 3D visualization of the WMH. The PVWMH mean width and mean intensity amplitudes were directly and highly correlated with each other $(\rho=0.55, P<.001)$ for the whole sample.

Subjects with PVWMH categorized as thin white lines exhibited expected thin widths $(3.2 \mathrm{~mm}, \mathrm{IQR}=2.63-3.78)$ and sharp intensity peaks (33.99\% increase from the adjacent lateral trough, $\mathrm{IQR}=23.46-44.74)$. Compared with the thin white line category, both width and intensity (mean increase with respect to the adjacent lateral trough) were significantly greater in both the thick $\operatorname{rim}(5.29 \mathrm{~mm}, \mathrm{IQR}=4.69-6.14 ; 53.16 \%, \mathrm{IQR}=40.15-66.64)$ and extended PVWMH (6.17 mm, IQR $=5.14-7.56 ; 56.22 \%$, $\mathrm{IQR}=44.26-71.53)$ categories. Subtle extended PVWMH exhibited multiple small-intensity peaks proximal to the ventricles and ill-defined boundaries of the PVWMH (as expected). This category was found to have a median width of $3.86 \mathrm{~mm}$ (IQR = $2.70-5.00 \mathrm{~mm}$ ) and a median intensity amplitude of $16.44 \%$ $(\mathrm{IQR}=10.18-21.16)$, the latter being significantly lower than all other categories (Fig 3).

The difference between the sampled intensity of the healthy white matter, equivalent to the mean intensity measured from the adjacent lateral trough (as in "Materials and Methods"), and the PVWMH rim was significantly smaller in the thin white line category compared with the thick rim (mean difference $=54.82 \%$ ) and extended PVWMH (mean difference $=68.31 \%$ ) categories. This means a gradual increase in the intensity of the PVWMH from the ventricular wall toward the deep white matter, with an increase in the load of white matter changes.

\section{Volumetric Analysis}

Overall, the widths of PVWMH were found to be highly correlated $(P<.001)$ with WMH volume, uncorrected $(\rho=0.61)$ and corrected by intracranial volume $(\rho=0.62)$. The WMH volume (corrected and uncorrected) was significantly correlated with the width of 3 PVWMH category groups separately without excluding outliers (ie, outside the 5\%-95\% range): thin white matter $(\rho=0.32, P<.001)$; thick rim $(\rho=0.61, P<.001)$; and extended PVWMH, $(\rho=0.44, P<.001)$, but not with the subtle extended PVWMH $(\rho=-0.20, P=.11)$. Similarly, an overall significant correlation $(P<.001)$ was found between mean intensity amplitude and WMH volume uncorrected $(\rho=0.45)$ and corrected by intracranial volume $(\rho=0.42)$ in all categories with the exception of the diffuse subtle extended PVWMH $(\rho=0.02, P=.84)$.

\section{Distributional Analysis}

Among 665 subjects, we found only 1 subject who exhibited a PVWMH width of $>13 \mathrm{~mm}(14.71 \mathrm{~mm})$. In the extended PVWMH category (mean PVWMH width $=6.52 \pm 0.2 \mathrm{~mm}$ ), $57 / 90$ conformed to $<7 \mathrm{~mm}$, and 71/90 to $<8 \mathrm{~mm}$, leaving only $19 / 90$ subjects within the sample for whom these distances may not be applicable.

The 3D representation of the WMH showed that subjects in the thin white line category had WMH that strictly adhered to the ventricle walls; however, regions of discrete DWMH were also present. In both the thin white line and thick rim categories, $\mathrm{PVWMH}$ were disconnected from hyperintensities in the deep 


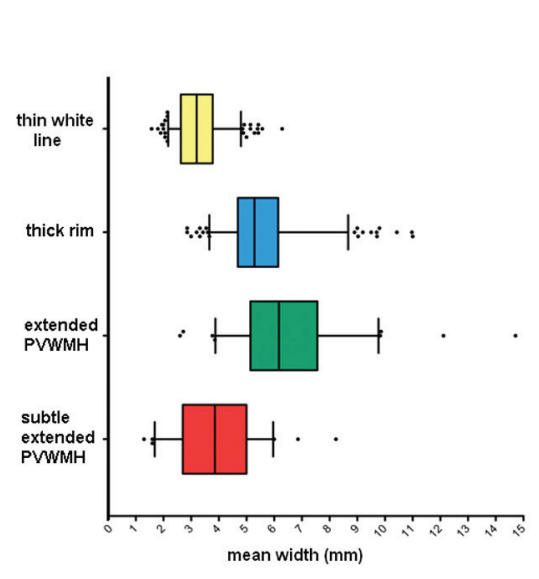

A

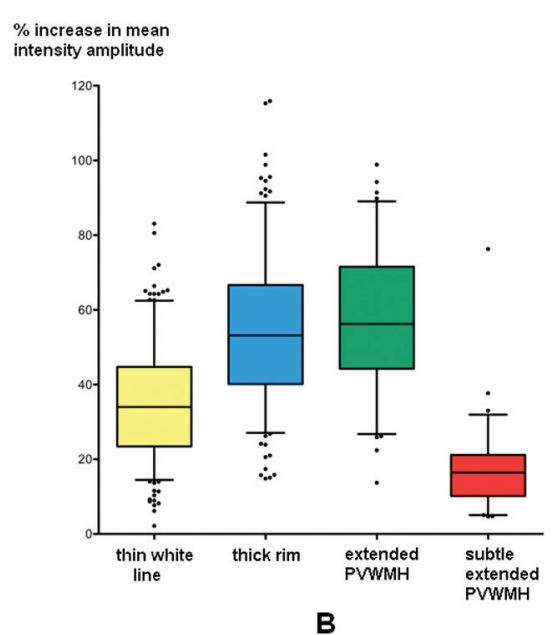

FIG 3. Box-and-whisker plots showing the distribution of PVWMH. Mean width $(A)$ and mean intensity amplitude (percentage peak from adjacent lateral trough) $(B)$ across the different categories. Boxes indicate $25 \%-75 \%$ interquartile range; horizontal lines in boxes, median width; whiskers, 5\%-95\% range; dots, detected outliers that reside outside the whisker range.
On the basis of vascular neuroanatomy, it has been suggested that the boundary of PVWMH and DWMH may lie within a 3- to 13-mm watershed zone. ${ }^{8,23}$ For our sample, the mean width of the PVWMH in the extended PVWMH category agreed with more specific suggestions that PVWMH may be separately identified from DWMH by distance thresholds ${ }^{4,9}$ of $<7$ or $<8 \mathrm{~mm}$. For scans on the third category (extended PVWMH), $8 \mathrm{~mm}$ also seemed an adequate distance for considering the boundary between PVWMH and DWMH. Previous investigations have found that volumetric measures of the ventricles, ${ }^{24}$ intracranial space, and brain tissue are highly variable between subjects. In this birth cohort, the use of WMH volume uncorrected or corrected either for brain tissue or intracranial volume has been demonstrated not to affect the analysis of white matter and subcortical regions, albeit the visual impression of continuity can be observed in 2D MR imaging. In the extended PVWMH category, extensive confluence was found between periventricular white matter intensities and DWMH. In this category, regions of nonconfluence were typically found lateral to the midventricular PVWMH as "pockets" of healthy white matter between periventricular white matter intensities and DWMH regions. 3D representation of the WMH of those subjects with diffuse subtle extended PVWMH revealed highly erratic and fragmented distributions of both PVWMH and DWMH (Fig 1).

\section{Relationship with Vascular Risk Factors}

A stepwise increase was found in the percentage of subjects falling within each of the first 3 categories (thin white line, thick rim, extended PVWMH) and their histories of hypertension, hypercholesterolemia, and evidence of stroke (Fig 4A). PVWMH were significantly wider in subjects with hypertension $(P=.03)$ and evidence of stroke $(P<.001)$, regardless of the category (Fig $4 B)$.

\section{DISCUSSION}

It has previously been suggested that PVWMH of $<2$ voxels wide are a manifestation of a partial volume effect ${ }^{5}$ or, alternatively, artifacts due to CSF pulsation. ${ }^{22}$ In this study, the PVWMH rim mean signal intensity in the thin white line that borders the ventricles was highly correlated and proportional with both total burden (volume) of WMH and the width of PVWMH (maximum of $3 \mathrm{~mm}$ for this category), as well as in the 2 categories for which such a result would be obvious: PVWMH penetrating into the deep white matter and large caps confluent with DWMH. An artifactual phase shift effect (produced by CSF pulsation) or truncation artifacts (common in areas where high and low signals are adjacent to each other) would not show these correlations. These findings suggest that these thin PVWMH are evidence of true white matter abnormalities. However, it does not mean that artifacts do not occur at the ventricle wall but, rather, that these artifacts cannot be identified by measurements of PVWMH width or signal intensity on FLAIR MR imaging.
WMH load ${ }^{20}$ as the results presented here corroborate.

Evidence of advanced leukoaraiosis on intensity analysis reveals a typical pattern (extended PVWMH) of a juxtaperiventricular signal-intensity peak followed by another DWMH peak. This pattern is also found on 3D distributional mapping, but only at the PVWMH rim do the surrounding caps of the frontal and occipital horns exhibit extensive confluence with DWMH. This commonly found trough of signal intensity at the PVWMH rim may mark the boundary of the periventricular white matter hyperintensities and DWMH distinction and may conform to a dichotomy on the basis of rules of continuity to the ventricle. ${ }^{6,7}$ This distinction, less informative than advanced distributional probabilistic atlases, has proved valid in clinical practice and research.

We found a positive and significant association between PVWMH width and hypertension, which is a small-vessel disease risk factor considered to be more highly associated with DWMH. ${ }^{2,5,25-27}$ However, an alternative interpretation of this finding is that higher widths are associated with higher total $\mathrm{WMH}$ volumes and thus are due to an indirect association, or that certain areas of PVWMH that penetrate into the deep WM should be considered DWMH. Evidence of subependymal defects, transudation of ventricular CSF, and impaired cerebral autoregulation in patients with hypertension who have severe PVWMH has been reported previously. ${ }^{28}$ We also found that PVWMH were significantly wider in subjects with evidence of stroke, but not in smokers, and in subjects with a history of hypercholesterolemia, diabetes, or cardiovascular disease. The percentage of subjects with hypertension, hypercholesterolemia, and evidence of stroke increased with increasing total WMH volume in thin, thick rim, and extended PVWMH categories.

The extensive presence of diffuse subtle PVWMH, also commonly termed "dirty-appearing white matter," has been rarely reported in the literature. Ge et $\mathrm{al}^{10}$ found that such regions, most often found in proximity to the ventricles, exhibit a signal greater than healthy white matter but lower than established WMH. 

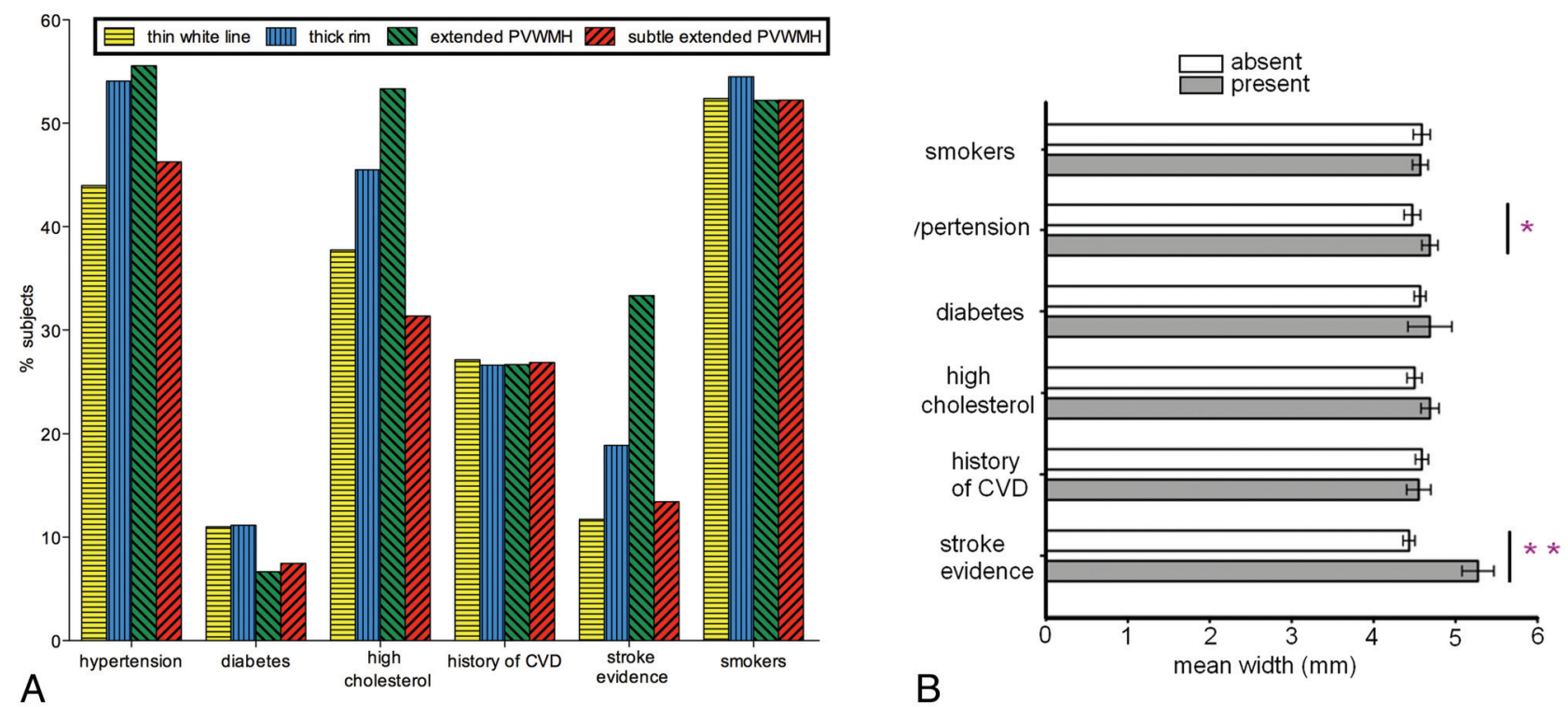

FIG 4. A, Percentage of subjects with vascular risk factors: hypertension, diabetes (type 1 and 2), hypercholesterolemia, smoking history, and history of cardio- and cerebrovascular disease. B, PVWMH mean width found in subjects with either the presence or absence of vascular risk factors. Mean width is significantly higher in those with hypertension $(+4.81 \%, P=.03)$ and evidence of stroke $(+18.97 \%, P<.001)$.

The current investigation confirms this finding in 67 subjects (10.08\%), with mean intensity amplitudes for these WMH significantly lower than those in other WMH categories and higher than those in healthy white matter. The underlying pathologic processes were not explored in this study, but the fact that no correlations were found between the width or intensity of this type of PVWMH and WMH total volume or the vascular risk factors explored in this study supports the suggestion that diffuse subtle extended PVWMH are evidence of evolving, preactive $\mathrm{WMH},{ }^{29}$ and/or secondary degenerative processes remote from established $\mathrm{WMH},{ }^{19}$ which can be confirmed on a follow-up scanning wave of these subjects. WMH segmentation and 3D distributional mapping confirm that these are ill-defined ${ }^{18}$ and fragmented regions.

The study strengths are that to our knowledge, this is the first study that jointly analyzes in detail the morphologic, distributional, volumetric, and intensity characteristics of PVWMH in an attempt to provide evidence about the nature (ie, real lesion or artifactual) of the periventricular thin hyperintense rim that commonly appears in brain FLAIR scans. A large sample size provided robust estimates of effect. Careful blinding of the analyses and a narrow age range to avoid confounding factors are also among the strengths of the study. Each conclusion resulted from a combination of multiple types of assessments, all conducted blinded to each other's results: visual (based on clinically proved visual rating scales and outcomes), computational, and clinical (vascular risk factors). The Intensity Analyzer tool presented is relatively fast, taking only $<5$ minutes to assess the width and intensity for a single subject (including the manual placing of the measuring line), and allows linear measurements, histogram, and voxelbased intensity analyses of tissues and anomalies on MR images. We used coregistered FLAIR and T2*-weighted images and multispectral data fusion to segment the $\mathrm{WMH} .{ }^{21}$ Combinations of multiple sequences have proved to largely remove the artifactual confound. $^{30,31}$
The study also has limitations. Despite the fact that visual assessment and categorization of PVWMH in "borderline" cases were discussed and agreed on between the 2 observers, the distinction from 1 category to another was not clear in some cases. Also, because WMH segmentations and volumes were not divided into periventricular white matter hyperintensities and DWMH as previous investigators have done, ${ }^{7,12}$ the associations of WMH volume with other parameters are not sensitive to differences between periventricular white matter hyperintensities and DWMH. Another limitation is that the data used in our analysis merely indicated whether the vascular risk factor was present or absent, not allowing detailed analyses of these variables (such as blood pressure measurement) as performed by other investigators. ${ }^{25,26}$

The analysis presented here used a semiquantitative PVWMH visual rating scale. Although the intra-/interobserver agreements on the categorization process were 0.99 and 0.97 , respectively, a fully automatic quantitative WMH mapping ${ }^{9,24}$ will allow a more detailed study of the WMH distribution. This study used data obtained from community-dwelling individuals. The distribution patterns of PVWMH categories and widths might differ in disease cohorts.

\section{Recommendations for Further Investigation}

Few studies have reported or addressed the pathologic correlates of periventricular white matter hyperintensities and DWMH. ${ }^{32}$ Further investigation is needed to identify reliable indicators of artifacts on brain MR imaging. Using pathologic samples may also allow the comparison of PVWMH on MR imaging with pathologic evidence of white matter abnormalities and may resolve the problem of the extent of PVWMH caused by artifacts. Referral to or combination with other sequences such as T1-, T2-, and proton attenuation-weighted MR imaging; the use of sequence coregistration; and multiple-level assessment of morphometric features $^{33}$ may otherwise guide the distinctions explored here (ie, 
between categories of PVWMH, PVWMH and DWMH, or thin white line and artifacts). Determining the significance of the fragmentation found in thin white line and diffuse PVWMH categories may also be achievable. These findings might indicate true white matter abnormalities and artifacts or might instead identify inaccuracies in segmentation (due to difficulties of definition from surrounding tissues ${ }^{19}$ ).

This study advocates considering the use of quantitative measures of intensity with other features like volume and distribution in the study of WMH. The methodology followed here, though not intended to make recommendations for the definitive ranges of PVWMH (collectively or in different categories), complements the qualitative assessment of WMH with quantitative measures of PVWMH width and intensity.

The $3 \mathrm{D}$ visualization techniques presented here and in other studies $^{7}$ should be explored further in studies of WMH to clarify the spatial distribution of WMH, enabling stronger conclusions about defining subtypes, etiology, and progression. This study supports the contention that $3 \mathrm{D}$ analysis avoids some of the limitations of $2 \mathrm{D}$ axial analysis, ${ }^{12}$ which allowed identifying otherwise undetected fragmented patterns of PVWMH.

\section{CONCLUSIONS}

PVWMH intensity levels, distribution, and association with risk factors and disease suggest that in old age, these are true tissue abnormalities and therefore should not be dismissed as artifacts. Dichotomizing periventricular white matter hyperintensities and DWMH by continuity from the ventricle edge toward the deep white matter is possible.

Disclosures: Maria del Carmen Valdés Hernández-RELATED: Grant: Age UK, * UK Medical Research Council, ${ }^{*}$ Comments: The work was funded as part of the project Disconnected Mind: LBC 1936 (http://www.disconnectedmind.ed.ac.uk/), UNRELATED: Employment: Row Fogo Charitable Trust.* Susana Muñoz ManiegaRELATED: Grant: Age UK, ${ }^{*}$ UK Medical Research Council, ${ }^{*}$ Comments: The work was funded by Age UK and the UK Medical Research Council as part of the project Disconnected Mind: LBC 1936 (http://www.disconnectedmind.ed.ac.uk/). Benjamin S. Aribisala—RELATED: Grant: Age UK, * UK Medical Research Council, ${ }^{*}$ Comments: The work was funded as part of the Disconnected Mind Project, UNRELATED: Employment: Medical Research Council.* Mark E. Bastin—RELATED: Grant: UK Medical Research Council.* lan J. Deary—RELATED: Grant: Age UK, * Medical Research Council,* Comments: Funds for cohort recruitment and brain imaging, UNRELATED: Board Membership: Medical Research Council, Comments: small sum for attending Neurosciences and Mental Health Board. Joanna M. Wardlaw-RELATED: Grant: Row Fogo Trust, ${ }^{*}$ Comments: academic grant to institution to support the work. *Money paid to the institution.

\section{REFERENCES}

1. Wen W, Sachdev P. The topography of white matter hyperintensities on brain MRI in healthy 60- to 64-year-old individuals. Neuroimage 2004;22:144-54

2. Fazekas F, Chawluk JB, Alavi A, et al. MR signal abnormalities at $\mathbf{1 . 5}$ T in Alzheimer's dementia and normal aging. AJR Am J Roentgenol 1987;149:351-56

3. Krishnan KR, Boyko OB, McDonald WM, et al. Magnetic-resonance morphometry: Image-analysis methodology development for affective disorder. Depression 1993;1:159-71

4. Brickman AM, Sneed JR, Provenzano FA, et al. Quantitative approaches for assessment of white matter hyperintensities in elderly populations. Psychiatry Res 2011;193:101-06

5. Payne ME, Fetzer DL, MacFall JR, et al. Development of a semiautomated method for quantification of MRI gray and white matter lesions in geriatric subjects. Psychiatry Res 2002;115:63-77
6. Kim KW, MacFall JR, Payne ME. Classification of white matter lesions on magnetic resonance imaging in elderly persons. Biol Psychiatry 2008;64:273-80

7. Ramirez J, Gibson E, Quddus A, et al. Lesion Explorer: a comprehensive segmentation and parcellation package to obtain regional volumetrics for subcortical hyperintensities and intracranial tissue. Neuroimage 2011;54:963-73

8. Sachdev P, Chen X, Wen W. White matter hyperintensities in midadult life. Curr Opin Psychiatry 2008;21:268-74

9. van der Lijn F, Verhaaren BF, Ikram MA, et al. Automated measurement of local white matter lesion volume. Neuroimage 2012;59: 3901-08

10. Ge Y, Grossman RI, Babb JS, et al. Dirty-appearing white matter in multiple sclerosis: volumetric MR imaging and magnetization transfer ratio histogram analysis. AJNR Am J Neuroradiol 2003; 24:1935-40

11. Appelman AP, Vincken KL, van der Graaf Y, et al. White matter lesions and lacunar infarcts are independently and differently associated with brain atrophy: the SMART-MR study. Cerebrovasc Dis 2010;29:28-35

12. DeCarli C, Fletcher E, Ramey V, et al. Anatomical mapping of white matter hyperintensities (WMH): exploring the relationships between periventricular WMH, deep WMH, and total WMH burden. Stroke 2005;36:50-55

13. Deary IJ, Gow AJ, Taylor MD, et al. The Lothian Birth Cohort 1936: a study to examine influences on cognitive ageing from age 11 to age 70 and beyond. BMC Geriatr 2007;7:28

14. Wardlaw JM, Bastin ME, Valdés Hernández MC, et al. Brain aging, cognition in youth and old age and vascular disease in the Lothian Birth Cohort 1936: rationale, design and methodology of the imaging protocol. Int J Stroke 2011;6:547-59

15. van Straaten EC, Fazekas F, Rostrup E, et al. Impact of white matter hyperintensities scoring method on correlations with clinical data: the LADIS study. Stroke 2006;37:836-40

16. Enzinger C, Fazekas F, Ropele S, et al. Progression of cerebral white matter lesions: clinical and radiological considerations. J Neurol Sci 2007;257:5-10

17. Conijn MM, Kloppenborg RP, Algra A, et al. Cerebral small vessel disease and risk of death, ischemic stroke, and cardiac complications in patients with atherosclerotic disease: the Second Manifestations of ARTerial disease-Magnetic Resonance (SMART-MR) study. Stroke 2011;42:3105-09

18. Moore GR, Laule C, Mackay A, et al. Dirty-appearing white matter in multiple sclerosis: preliminary observations of myelin phospholipid and axonal loss. J Neurol 2008;255:1802-12, discussion 1812

19. Filippi M, Rocca MA. Dirty-appearing white matter: a disregarded entity in multiple sclerosis. AJNR Am J Neuroradiol 2010;31:90-91

20. Valdés Hernández MC, Morris Z, Dickie DA, et al. Close correlation between quantitative and qualitative assessments of white matter lesions. Neuroepidemiology 2013;40:13-22

21. Hernández MC, Ferguson KJ, Chappell FM, et al. New multispectral MRI data fusion technique for white matter lesion segmentation: method and comparison with thresholding in FLAIR images. Eur Radiol 2010;20:1684-91

22. Bakshi R, Caruthers SD, Janardhan V, et al. Intraventricular CSF pulsation artifact on fast fluid-attenuated inversion-recovery $\mathrm{MR}$ images: analysis of 100 consecutive normal studies. AJNR Am JNeuroradiol 2000;21:503-08

23. Mayer PL, Kier EL. The controversy of the periventricular white matter circulation: a review of the anatomic literature. AJNR Am J Neuroradiol 1991;12:223-28

24. Jongen C, van der Grond J, Anbeek P, et al. Construction of periventricular white matter hyperintensity maps by spatial normalization of the lateral ventricles. Hum Brain Mapp 2009;30:2056-62

25. Murray AD, Staff RT, Shenkin SD, et al. Brain white matter hyperintensities: relative importance of vascular risk factors in nondemented elderly people. Radiology 2005;237:251-57

26. Godin O, Tzourio C, Maillard P, et al. Antihypertensive treatment 
and change in blood pressure are associated with the progression of white matter lesion volumes: the Three-City (3C)-Dijon Magnetic Resonance Imaging Study. Circulation 2011;123:266-73

27. de Leeuw FE, de Groot JC, Oudkerk M, et al. Hypertension and cerebral white matter lesions in a prospective cohort study. Brain 2002;125:765-72

28. Pantoni L, Garcia JH. Pathogenesis of leukoaraiosis: a review. Stroke 1997;28:652-59

29. De Groot CJ, Bergers E, Kamphorst W, et al. Post-mortem MRIguided sampling of multiple sclerosis brain lesions: increased yield of active demyelinating and (p) reactive lesions. Brain 2001; 124:1635-45

30. Admiraal-Behloul F, van den Heuvel DM, Olofsen H, et al. Fully automatic segmentation of white matter hyperintensities in MR images of the elderly. Neuroimage 2005;28:607-17

31. Klöppel S, Abdulkadir A, Hadjidemetriou S, et al. A comparison of different automated methods for the detection of white matter lesions in MRI data. Neuroimage 2011;57:416-22

32. McAleese KE, Firbank M, Hunter D, et al. Magnetic resonance imaging of fixed post mortem brains reliably reflects subcortical vascular pathology of frontal, parietal and occipital white matter. Neuropathol Appl Neurobiol 2013;39:485-97

33. Scully M, Anderson B, Lane T, et al. An automated method for segmenting white matter lesions through multi-level morphometric feature classification with application to lupus. Front Hum Neurosci 2010;4:27 